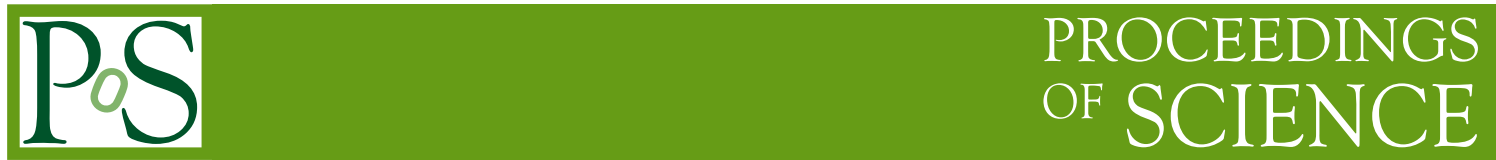

\title{
Ion-Channeling in Direct Dark Matter Crystalline Detectors
}

\section{Graciela GELMINI*†}

Dept. of Physics and Astronomy, University of California-Los Angeles

E-mail: gelmini@physics.ucla.edu

The channeling of the recoiling nucleus in crystalline detectors after a WIMP collision would produce a larger scintillation or ionization signal in direct detection experiments than otherwise expected. I present estimates of the importance of this effect in $\mathrm{NaI}, \mathrm{Si}$ and Ge crystals, using analytic models developed from the 1960's onwards to describe channeling and blocking effects.

Identification of Dark Matter 2010

July 26 - 302010

University of Montpellier 2, Montpellier, France

\footnotetext{
* Speaker.

${ }^{\dagger}$ Talk based on work done with Nassim Bozorgnia and Paolo Gondolo.
} 
Channeling and blocking in crystals refer to the orientation dependence of ion penetration in crystals. "Channeling" occurs when ions incident upon a crystal along symmetry axes and planes suffer a series of small-angle scatterings that maintain them in the open "channels" in between rows or planes of lattice atoms and thus penetrate much further than non-channeled ions. Channeled ions do not get close to lattice sites, where they would suffer a large-angle scattering which would take them out of the channel. "Blocking" is the reduction of the flux of ions originating in lattice sites along symmetry axes and planes due to the shadowing effect of the lattice atoms directly in front of the emitting lattice site (see e.g. the review by D. Gemmell [1] and references therein).

Channeling and blocking effects in crystals are extensively used in crystallography, in measurements of short nuclear lifetimes, in the production of polarized beams etc. Channeling must be avoided in the implantation of B, P and As atoms in Si crystals to make circuits. The channeling effect in $\mathrm{NaI}(\mathrm{Tl})$ crystals was first observed by Altman et al. [2] in 1973 who observed that channelled ions produce more scintillation light because they loose practically all their energy via electronic stopping rather than nuclear stopping. When ions recoiling after a collision with a WIMP move along crystal axes and planes, they give all their energy to electrons, so the quenching factor $Q$ (the fraction of the energy deposited in a collision that goes into scintillation or ionization) is $Q \simeq 1$ instead of e.g. $Q_{I} \simeq 0.09$ and $Q_{N a} \simeq 0.3$ in NaI(Tl). The potential importance of this effect for direct dark matter detection was first pointed out for NaI(Tl) by Drobyshevski in 2007 and soon after by the DAMA collaboration [3]. This last paper gave an estimate of the channeling fraction of recoiling ions as function of the energy, in which the fraction grew with decreasing energy to be $\simeq 1$ close to $1 \mathrm{keV}$. With this channeling fraction, the region of light WIMPs compatible with producing an annual modulation as measured by the DAMA collaboration shifted considerably to lower cross sections, by about one order of magnitude (see e.g. [4]). Channeling could also produce a novel dark matter signature. Since the WIMP wind comes preferentially from the direction fixed to the galaxy towards which the Sun moves, Earth's daily rotation makes this direction change with respect to the crystal, which could produce a daily modulation in the measured energy (equivalent to a modulation of the quenching factor). As first pointed out by Avignone, Creswick, Nussinov in 2008 [5], this modulation depends on the orientation of the crystal with respect to the galaxy, thus it would be a background free dark matter signature.

Our calculations [6] of the fraction of recoils that are channeled as function of recoil energy and direction use classical analytic models which started to be developed in the 60's and 70's, soon after channeling was discovered, for ions of energy $\mathrm{MeV}$ and higher. We use in particular Lindhard's model [7], supplemented by the planar channel models of Morgan and Van Vliet [8] and the 1995-1996 work of G. Hobler [9] on low energy channeling (applied to ion implantation in $\mathrm{Si}$ ). In these models the rows and planes of lattice atoms are replaced by continuum strings and planes in which the screened Thomas-Fermi potential is averaged over a direction parallel to a row or plane of lattice atoms to find the continuous potential $U$ (see Fig. 1.a for examples of $U$ ). The appearance of continuous strings or planes can be understood as the overlap of the "Coulomb shadows" of individual atoms in a lattice row or plane behind the direction of arrival of a parallel beam of positive ions, when the beam arrives at a small enough angle, smaller than a critical angle $\psi_{c}$. Then, the individual shadows overlap forming a string or plane of thickness $\rho_{c}$ within which the scattered ions do not penetrate (see e.g. Fig. 4.6 of Ref. [10]). We included just one string or plane, which is a good approximation except when $\rho_{c}$ approaches the radius of the channel. 

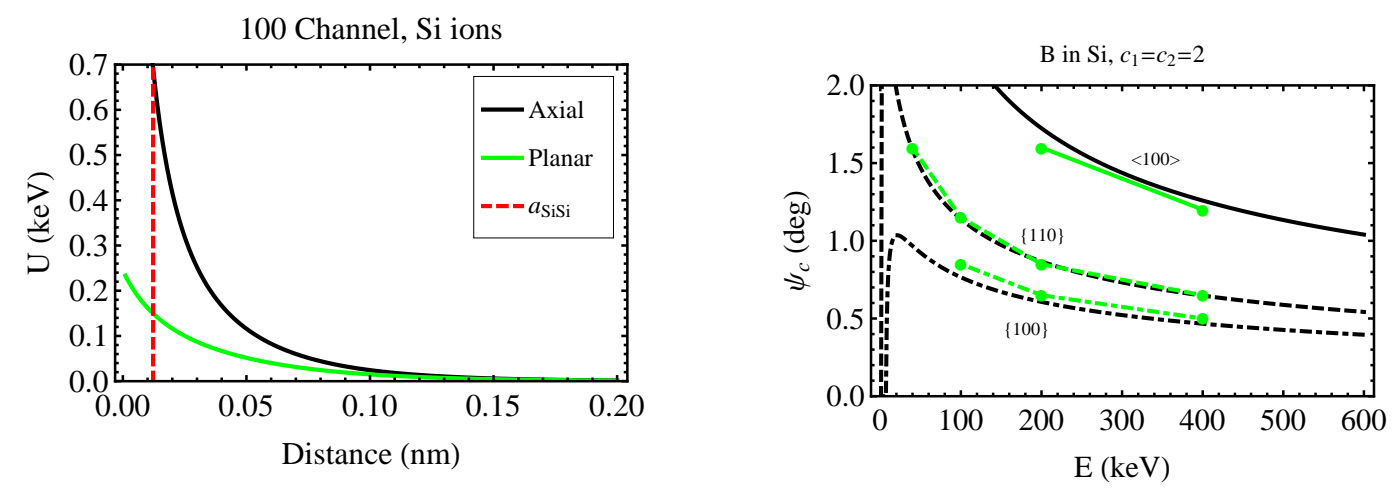

Figure 1: a. (left) Continuum potential for a string (black) and plane (green) for a $\mathrm{Si}$ ion propagating in $\mathrm{Si}$ as function of the distance perpendicular to the string or plane. b. (right) Oberved (green) and predicted with $c=2$ (black) channeling angles for $\mathrm{B}$ ions propagating in $\mathrm{Si}$ at room $\mathrm{T}$.

Lindhard proved that for channeled ions the "transverse energy" $E_{\text {perp }}=M v_{\text {perp }}^{2} / 2+U \simeq$ $E \phi^{2}+U$ is conserved, where $M, E=M \mathrm{v}^{2} / 2$ are the mass and kinetic energy of the propagating ion, $\mathrm{v}_{\text {perp }}=\mathrm{v} \sin \phi \simeq \mathrm{v} \phi$ is the component of the velocity perpendicular to the string or plane and $U$ is the continuum axial or planar potential at the position of the ion. Thus, for a particular $E_{\text {perp }}$ (determined by the recoil energy $E$, initial recoil angle $\phi_{i}$ and initial potential and $U_{i}$ ), a channeled propagating ion does not approach the string or plane closer than a minimum distance $\rho_{\text {min }}$ (for which $\mathrm{v}_{\text {perp }}=0$ ) and far away from the string or plane, close to the middle of the channel, the ion moves on a trajectory forming an angle $\psi$ with the string or plane, given by $E_{\text {perp }}=E \phi_{i}^{2}+U_{i}=U\left(\rho_{\text {min }}\right)=E \psi^{2}+U_{\text {middle. }}$. Channeling occurs when $\rho_{\text {min }}>\rho_{c}$, which amounts to $\psi=\sqrt{\left.\left[U\left(\rho_{\text {min }}\right)-U_{\text {middle }}\right)\right] / E} \leq \psi_{c}=\sqrt{\left.\left[U\left(\rho_{c}\right)-U_{\text {middle }}\right)\right] / E}$.

All the difficulty of this approach resides in calculating $\rho_{c}$. Including temperature $(T)$ effects, the critical distance can be approximated by $\rho_{c}(E, T)=\sqrt{\rho_{c}^{2}(E)+\left[c u_{1}(T)\right]^{2}}$, where $\rho_{c}(E)$ is the critical distance for a perfectly rigid lattice (which decreases as $E$ increases, as shown in Fig. 2.a), $u_{1}(T)$ is the 1-dimensional amplitude of thermal fluctuations (we used the Debye model) which increases with $T$, and $c$ is a dimensionless number which (for several crystals and propagating ions, different than the ones we study) was found through data and simulations to be $1<c<2$. At large enough energies $\rho_{c}(E, T) \simeq c u_{1}(T)$ and thus as $T$ increases the strings are planes become thicker, the channels narrower and $\psi_{c}$ smaller (see Fig. 2). Using this formalism with $c=2$ we could reproduce data on channeling angles of $\mathrm{B}$ and $\mathrm{P}$ ions in Si measured at room $T$ provided by Hobler [9] (shown in green for B in Fig.1.b). As shown in Figs. 1.b and 2.b the critical angle $\psi_{c}$ increases with decreasing energy until the critical distance for channeling $\rho_{c}$ approaches the radius of the channel, at which point $\psi_{c}$ goes zero (see Fig. 1.a). At still lower energies, $\rho_{c}$ should be larger than the radius of the channel, what indicates that channeling is not possible (thus $\psi_{c}=0$ ).

The channeling of an ion depends not only on the initial angle its trajectory makes with a string or plane, but also on its initial position. The DAMA collaboration [3] calculated the channeling fraction for ions starting their motion close to the middle of the channel, where channeling happens if $\psi<\psi_{c}$. However this is not the case in dark matter detectors, since the recoiling ions start their motion at or close to their original lattice sites (and leave those sites empty). For 

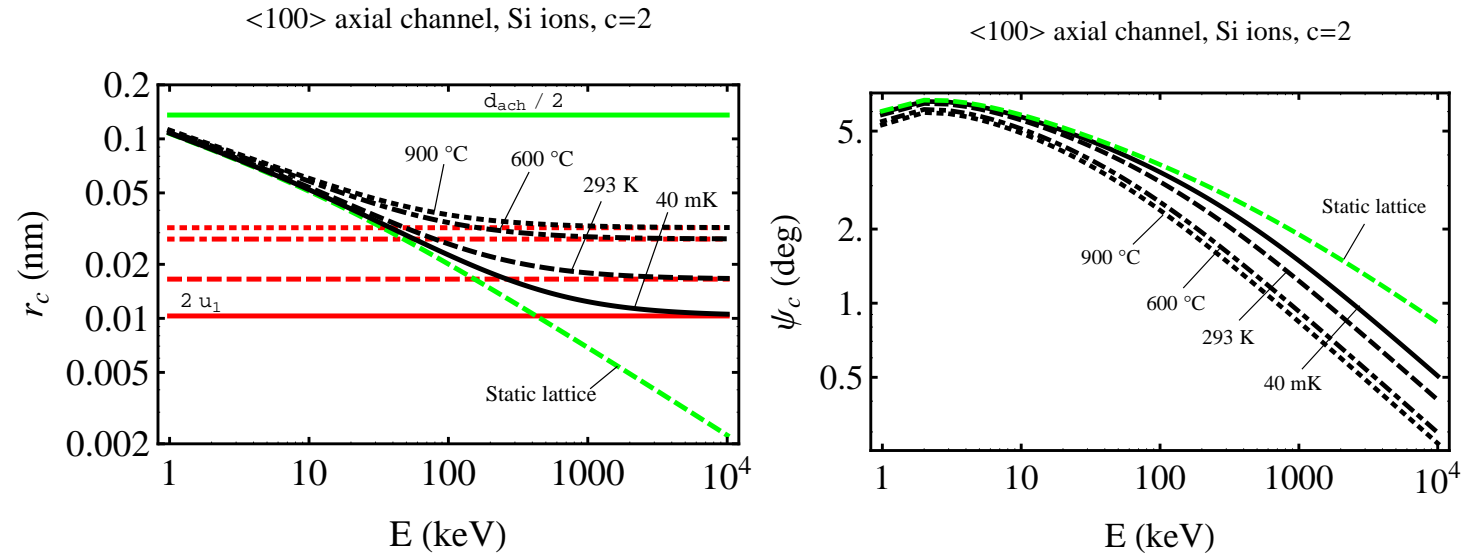

Figure 2: a. (left) Critical channeling distance $r_{c}$ and b. (right) critical channeling angles $\psi_{c}$ for a Si ion propagating in the $\langle 100\rangle$ channel of a Si crystal, for a static lattice (dashed green) and $T$ corrected (solid black lines) at different $T$ with $c=2\left(2 u_{1}\right.$ shown in red and the radius of the channel in solid green)
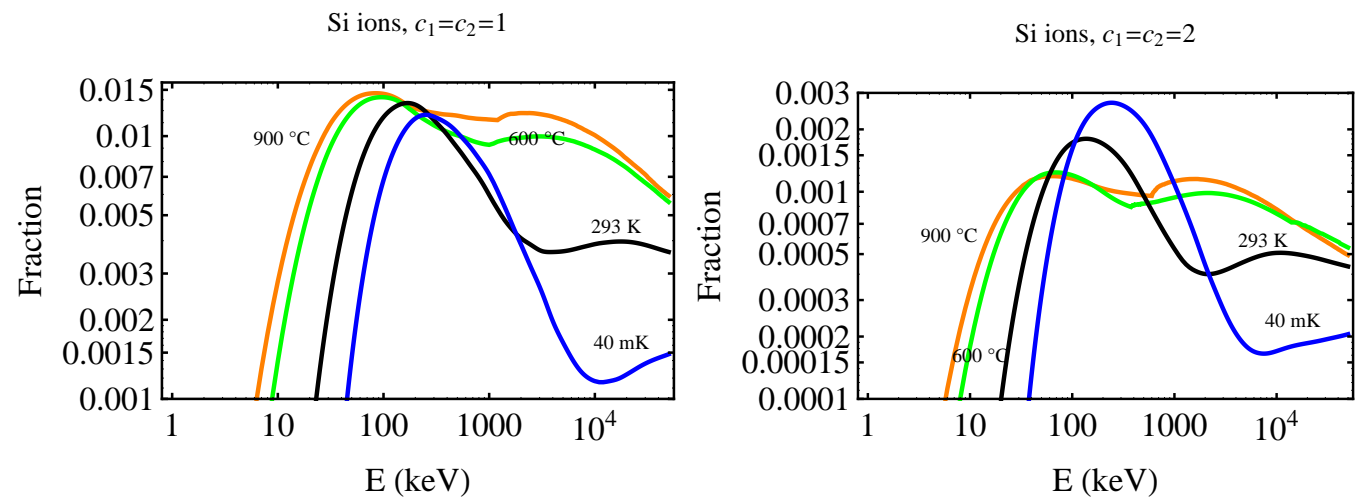

Figure 3: Channeling fractions for a Si ions ejected from lattice sites in a Si crystal as function of the ion energy for different temperatures $T$ and $T$ effects computed with a. (left) $c=1$ and b. (right) $c=2$.
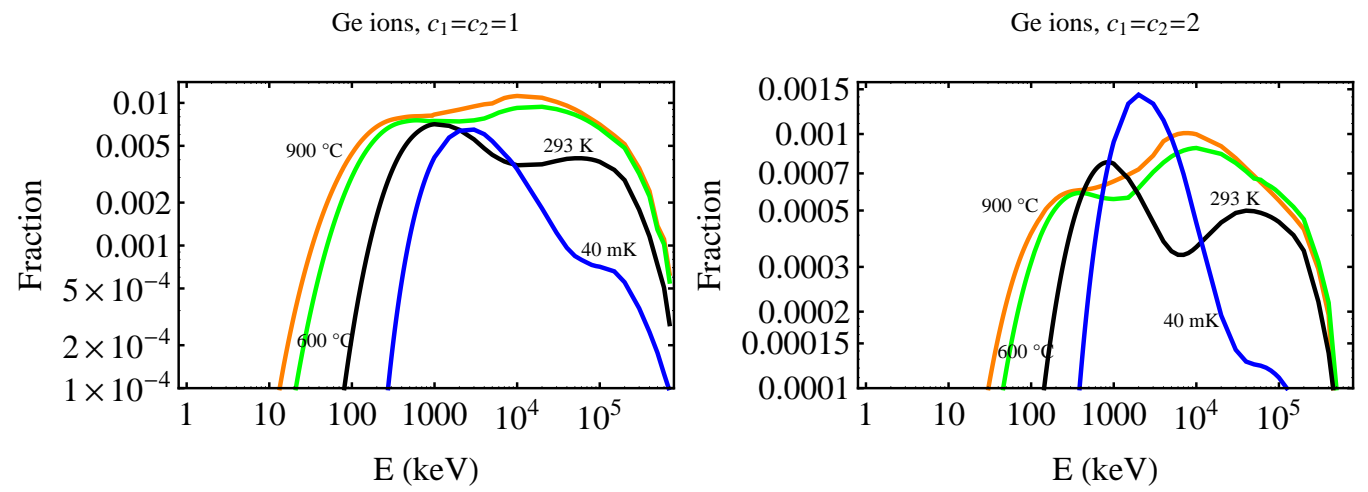

Figure 4: Same as Fig. 3 but for Ge ions ejected from lattice sites in a Ge crystal. 

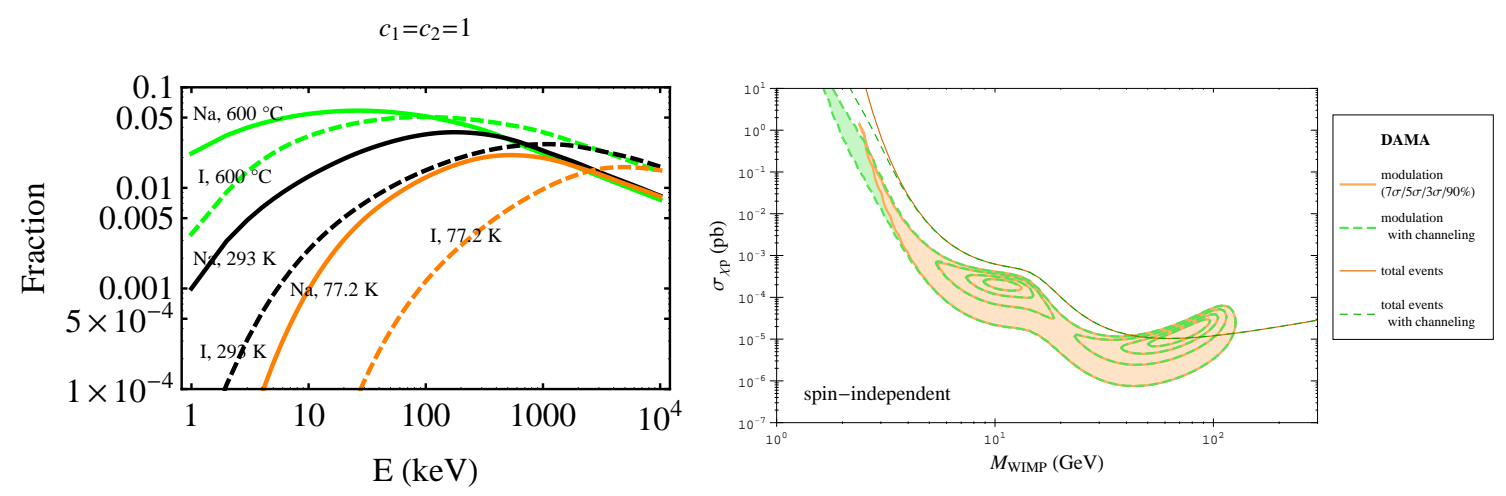

Figure 5: a. (left) Upper bounds to the channeling fraction of $\mathrm{Na}$ and I ions ejected from lattice sites in a NaI crystal for several temperatures (with $c=1$ ). b. (right) Using the $\mathrm{T}=293 \mathrm{~K}$ curve as the channeling fraction in the DAMA detectors the region of compatibility with the DAMA signal for WIMPs with spin-independent interaction is the same with and without channeling at less than $7 \sigma$ [12].

recoiling ions, blocking effects (neglected in the DAMA calculation) are important. In fact in a perfect lattice no recoiling ion would be channeled (because of what Lindhard called the "rule of reversibility"), but due to lattice vibrations the collision with a WIMP may happen while an atom is displaced with respect to the string or plane where it belongs. If it is initially far enough it may be channeled. For an axial channel, the probability distribution function of the perpendicular distance to the row of the colliding atom due to thermal vibrations can be represented by a Gaussian, $g(\rho)=\left(\rho / u_{1}^{2}\right) e^{\left(-\rho^{2} / 2 u_{1}^{2}\right)}$. Thus, in our model the probability that an ion is channeled is given by the fraction of nuclei which can be found at a distance larger than a minimum distance $\rho_{i, \text { min }}$ (determined by $\rho_{c}$ and the initial recoil angle) from the string at the moment of collision, $P_{C h}=\int_{\rho_{i, \min }}^{\infty} \operatorname{drg}(\rho)=e^{\left(-\rho_{i, \min }^{2} / 2 u_{1}^{2}\right)}$. Notice that any uncertainty in $\rho_{c}$ is exponentially enhanced in the channeling fraction. Similar equations apply to planar channels. To obtain the total geometrical channeling fraction, we average the channeling probability over initial recoil directions, assuming they are isotropically distributed. We do it numerically by performing a Riemann sum once the sphere of directions has been divided using a Hierarchical Equal Area iso-Latitude Pixelization (HEALPix) [11] (a novel use for HEALPix).

The channeling fractions we obtain are strongly $T$ dependent (see e.g. Figs. 2,3 and 4.a). As $T$ increases the probability of finding atoms far from their equilibrium lattice sites increases, which increases the channeling fractions, but the critical distances $\rho_{c}$ become larger $\left(\simeq c u_{1}\right.$ at large enough energies) which decreases the channeling fractions. The fractions are smaller for larger values of $c$, as can be seen for Si and Ge in Fig. 3 and 4. We expect the fractions to be in between those for $c=1$ and those for $c=2$. Moreover, we have not included any dechanneling effects due to impurities or dopants. So the fractions we produced must be considered upper bounds. This is even more so for $\mathrm{NaI}$ and $\mathrm{CsI}$, for which we have not found data equivalent to those provided by Hobler for $\mathrm{Si}$ (useful for $\mathrm{Ge}$ too, which has the same structure of $\mathrm{Si}$ ) so we can provide upper bounds on the channeling fractions instead of estimates, even forgetting about dechanneling effects. Upper bounds on channeling fractions in NaI are shown in Fig. 4.a for $c=1$ and several temperatures. Using the fractions in Fig. 5.a corresponding to the temperature of the DAMA/NaI and the DAMA/LIBRA experiments, $\mathrm{T}=293 \mathrm{~K}$, the difference in the WIMP region corresponding 
to the DAMA annual modulation signal differs only at $7 \sigma$ is channeling is included or not included (as can be seen in Fig. 5.b taken from Ref. [12]).

To conclude, the effect of blocking is important to understand the channeling of ions ejected from lattice sites by interactions with dark matter particles. Analytic models give good qualitative results but channeling data and or simulations are necessary to get reliable quantitative results. Finally, the daily modulation of the scintillation or ionization in crystalline detectors due to channeling, a potentially background free dark matter signal, deserves further investigation.

\section{References}

[1] D. S. Gemmell, Channeling And Related Effects In The Motion Of Charged Particles Through Crystals, Rev. Mod. Phys. 46129 (1974).

[2] R. Altman, H. B. Dietrich, R. B. Murray, and T. J. Rock, Scintillation Response of NaI(T1) and KI(T1) to Channeled Ions, Phys. Rev. B 7, 1743 (1973).

[3] R. Bernabei et al., Possible implications of the channeling effect in NaI(Tl) crystals, Eur. Phys. J. C 53, 205 (2008) [arXiv:0710.0288 [astro-ph]].

[4] C. Savage, G. Gelmini, P. Gondolo and K. Freese, Compatibility of DAMA/LIBRA dark matter detection with other searches, JCAP 0904, 010 (2009) [arXiv:0808.3607 [astro-ph]].

[5] F. Avignone, R. Creswick and S. Nussinov, Searching direction dependent daily modulation in dark matter detectors, arXiv:0807.3758 [hep-ph]; R. Creswick, S. Nussinov and F. Avignone, Directional Dependence and Diurnal Modulation in Dark Matter Detectors arXiv:1007.0214 [astro-ph.IM].

[6] N. Bozorgnia, G. B. Gelmini and P. Gondolo, Channeling in direct dark matter detection I: channeling fraction in NaI (Tl) crystals JCAP 1011, 019 (2010) [arXiv:1006.3110 [astro-ph.CO]]; Channeling in direct dark matter detection II: channeling fraction in Si and Ge crystals, JCAP 1011, 028 (2010) [arXiv:1008.3676 [astro-ph.CO]]; Channeling in direct dark matter detection III: channeling fraction in CSI crystals, JCAP 1011, 029 (2010) [arXiv:1009.3325 [astro-ph.CO]]; Channeling in direct dark matter detection V: channeling fraction in solid Xe, Ar and Ne arXiv:1011.6006 [astro-ph.CO].

[7] J. Lindhard, Influence of Crystal Lattice on the Motion of Energetic Charged Particles, Kongel. Dan. Vidensk. Selsk., Mat.-Fys. Medd. 34 No. 14 (1965).

[8] D. V. Morgan and D. Van Vliet, Computer studies of fast ion trajectories in crystals, Can. J. Phys. 46, 503 (1968); D. V. Morgan and D. Van Vliet, Critical approach distances and critical angles for channelling, Radiat. Effects and Defects in Solids 8, 51 (1971).

[9] G. Hobler, Critical angles and low-energy limits to ion channeling in silicon, Radiation effects and defects in solids 139, 21 (1996); G. Hobler, Theoretical estimate of the low-energy limit to ion channeling, Nucl. Instrum. Methods Phys. Research (NIM), B 115, 323 (1996).

[10] H. Kudo, Ion Channeling and High-Energy Shadowing, chapter 4 in Ion-induced Electron Emission from Crystalline Solids, Springer Tracts in Modern Physics, 2002.

[11] K. M. Górski et al., HEALPIX - a Framework for High Resolution Discretization, and Fast Analysis of Data Distributed on the Sphere, ApJ 622756 (2005).

[12] C. Savage, G. Gelmini, P. Gondolo and K. Freese, XENON10/100 dark matter constraints in comparison with CoGeNT and DAMA: examining the Leff dependence arXiv:1006.0972 [astro-ph.CO]. 